\title{
Implementación de las tareas semanales mediante la plataforma PoliformaT para la mejora de resultados en el aprendizaje por proyectos.
}

\author{
Sergi Montava Jordàa, Samuel Sanchez Caballero ${ }^{b}$, Miguel Ángel Sellés Cantó ${ }^{\mathbf{y}} \mathbf{y}$ \\ Antonio Vicente Martinez Sanz . \\ Departamento de Ingeniería Mecánica y de Materiales, Escuela Politécnica Superior de Alcoy. \\ Universitat Politècnica de València (sermonjo@mcm.upv.es ${ }^{\mathrm{a}}$; \\ maselles@dimm.upv.es ${ }^{\mathrm{c}}$; anmarsan@mcm.upv.es ${ }^{\mathrm{d}}$ )
}

\begin{abstract}
In this paper both the initiative and the results of the same in teaching innovation are presented, which consisted in the realization of exercises or weekly self-evaluable tests through the PoliformaT platform of the UPV, in the practices of the subject Theory and Design of Machines to reinforce and strengthen concepts. The result of the implementation of this initiative has been positive for several aspects: the first of them has been to improve the results of the qualifications in the projects of the practices in its first year lunched and the second to obtain a direct relationship between the average note obtained from these exercises with the note of the projects.
\end{abstract}

Keywords: Project learning (PBL), Machine design, Weekly tasks, PoliformaT, Teaching innovation

\section{Resumen}

En el presente trabajo se presentan tanto la iniciativa como los resultados de la misma en innovación docente, la cual consistía en la realización de ejercicios o pruebas semanales auto evaluables a través de la plataforma PoliformaT de la UPV, en las prácticas de la asignatura Teoría y Diseño de Máquinas para reforzar y afianzar conceptos. El resultado de la implantación de esta iniciativa ha sido positiva por varios aspectos: el primero de ellos ha sido el conseguir mejorar los resultados de las calificaciones en los proyectos de las prácticas en su primer año de implantación y el segundo por obtener una relación directa entre la nota media conseguida de estos ejercicios con la nota de los proyectos. 
Palabras clave: Aprendizaje por proyectos (ABP), diseño de máquinas, tareas semanales, PoliformaT, Innovación docente

\section{Introducción}

La asignatura Teoría y Diseño de Máquinas (en adelante TDM) en la que se centra este trabajo es de carácter obligatorio y anual del tercer curso del Grado de Ingeniería Mecánica de la Universitat Politècnica de València. Según el plan de estudios actual (BOE 16-032011) consta de un total de 9,0 créditos ETCS (European Credits Transfer System) repartidos en 4,5 créditos para la parte teórica y 4,5 créditos para la práctica, por tanto, para poder superar la asignatura son igual de importantes ambas partes según SÁNCHEZ CABALLERO, SELLES CANTÓ, PLÁ FERRANDO, and MONTAVA JORDÀ (2018)

Durante varios cursos impartiendo las prácticas de la asignatura TDM mediante el Aprendizaje Basado en Proyectos (en adelante ABP) los resultados obtenidos no han sido los esperados por los profesores aumentando considerablemente el porcentaje de suspensos. Se detecto que los conceptos necesarios para la realización de los proyectos de Diseño de Máquinas no se alcanzaban solamente con las clases magistrales ni las prácticas de laboratorio. Tras un intento de mejorar esta deficiencia en el aprendizaje de conceptos se planteo la realización de diversas actividades optativas de refuerzo. A pesar de suponer un trabajo adicional tanto para los alumnos como para los profesores, se observo una mejora considerable en las calificaciones de los alumnos que las realizaron estas actividades. Entonces se pensó en la posibilidad de aplicar esta mejora a través tareas semanales auto evaluables con la plataforma PoliformaT de la UPV, viendo además los resultados de experiencias similares respecto a la autoevaluación.

Tal como indica LLORENS MOLINA (2017), la autoevaluación contribuye a la mejora del aprendizaje a través de cuatro clases de aportaciones:

- Pedagógicas, dado que la retroalimentación proporciona oportunidades para el cambio conceptual y la profundización en los contenidos, así como para adquirir nuevas habilidades y recursos metodológicos.

- Metacognitivas, al contribuir a una mayor consciencia por parte de estudiante acerca de sus carencias y limitaciones, potencialidades y evolución de su aprendizaje.

- De carácter práctico, ya que si se le asigna también una función sumativa, supone un importante ahorro de tiempo para el profesorado, sobre todo cuando imparte docencia a grandes grupo.

- Afectivas, ya que el alumno percibe la evaluación como un instrumento de aprendizaje más que como un elemento meramente sancionador de sus logros

\section{Objetivos}

El objetivo principal de este trabajo es mejorar o afianzar los conceptos principales necesarios para la realización de los proyectos de prácticas de la asignatura TDM y mejorar 
los resultados de estos, partiendo de la metodología de aprender haciendo (learning by doing) y la autoevaluación aplicada al ABP.

A partir de este objetivo principal surgen otros objetivos específicos como:

- Utilizar conocimientos de diversas áreas o de ejercicios anteriores para la resolución de proyectos más complejos.

- Aprendizaje y trabajo autónomo, con las pruebas semanales se alcanzan poco a poco los conociemientos necesarios.

- Auto evaluación, al tratarse de pruebas auto evaluables de forma inmediata los propios alumnos son capaces de detectar sus errores para poder corregirlos.

\section{Desarrollo de la innovación}

\subsection{Análisis de las causas}

Tras la implantación del ADP en las prácticas de TDM se aprecio un crecimiento considerable del nivel de suspensos de los trabajos de ambos cuatrimestres. Como se puede apreciar en la Figura 1 el porcentaje de los que suspenden el trabajo en el curso 2016/2017 estaba alcanzando valores entre el $42 \%$ y el 51\% para los trabajos del cuatrimestre A y del cuatrimestre B respectivamente. Estos valores fueron los que nos hicieron pensar que no era suficiente la realización de estos ejercicios optativos, ya que dada la baja participación en su realización solo mejoraban una pequeña parte de los alumnos que presentaban los trabajos. Por tanto, casi la mitad de los alumnos que presentaban los trabajos tenían que recuperar el trabajo o ir al examen final a recuperar el apartado de prácticas.

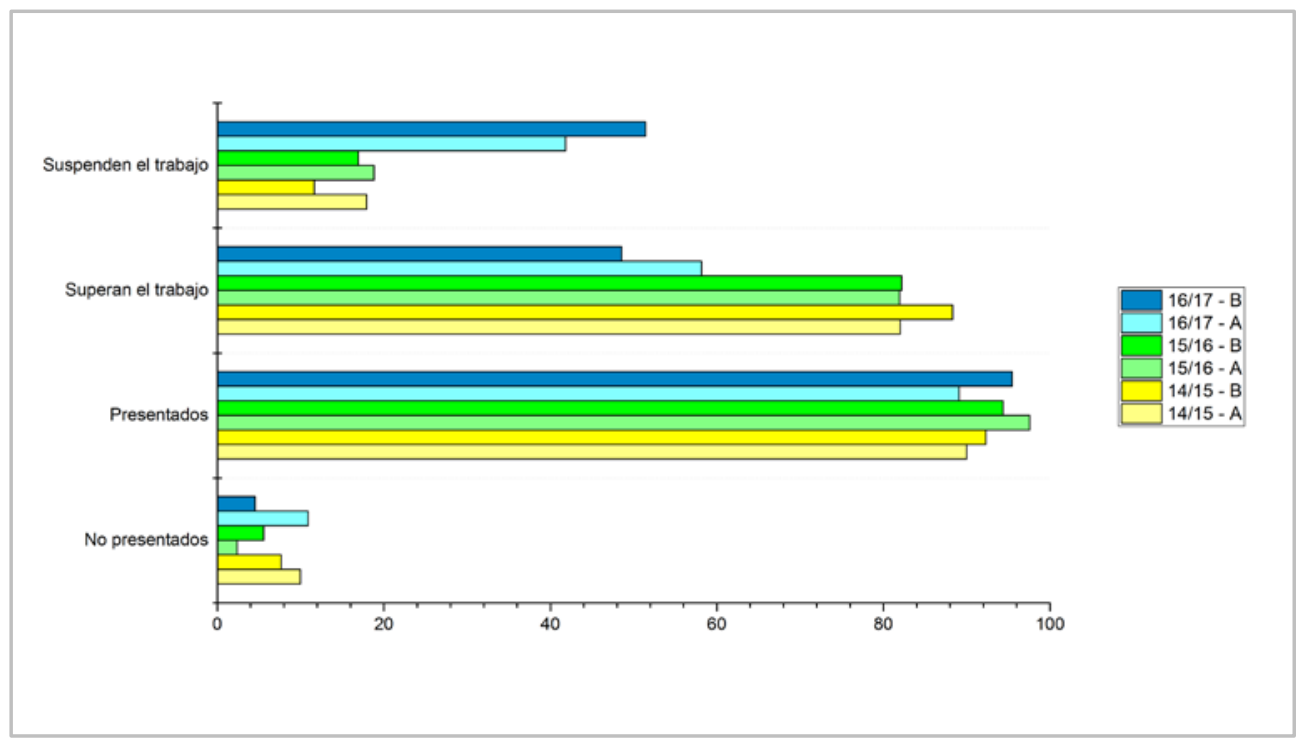

Fig. 1 Evolución presentación y entrega de los trabajos de prácticas en los últimos cursos 


\subsection{Estrategia de mejora}

Se realizo una pequeña reflexión entre los profesores implicados y tras realizar un pequeño estudio al respecto se observo que era una tendencia bastante común en la actualidad y habían surgido varios artículos de investigación docente al respecto como los de: LEÓN DE MORA, MOLINA CANTERO, MOLINA CANTERO, and BISCARRI TRIVIÑO (2009), CODESAL SANABRIA (2008), RAMÓN FERNÁNDEZ (2011) o MORÁN GRACIA and SEPÚLVEDA PINAR (2009).

La decisión fue el que estos ejercicios formasen parte de la nota final de asignatura, como un nuevo ítem PARTICIPACIÓN EN CLASE, en donde dependiendo de la contribución activa del alumno en la asignatura se conseguirá hasta un 10\% de esta. Para el siguiente curso 2017/2018 se procedió a reflejar esta mejora en la propia guía docente de la asignatura esperando que se obtuviesen resultados mejores a los del curso 2016/2017.

\subsection{Ejercicios Semanales}

Se tuvo que realizar una nueva programación de los ejercicios con un total de 12 ejercicios, divididos del E01 al E06 para el cuatrimestre A y del E07 al E12 para el cuatrimestre B. Estos ejercicios estaban disponibles para los alumnos en una carpeta denominada 02_EJERCICIOS desde el apartado de RECURSOS del PoliformaT de la asignatura TDM en formato pdf, así como cualquier anexo necesario, desde la fecha de su publicación los lunes de cada semana que había que realizar un ejercicio.

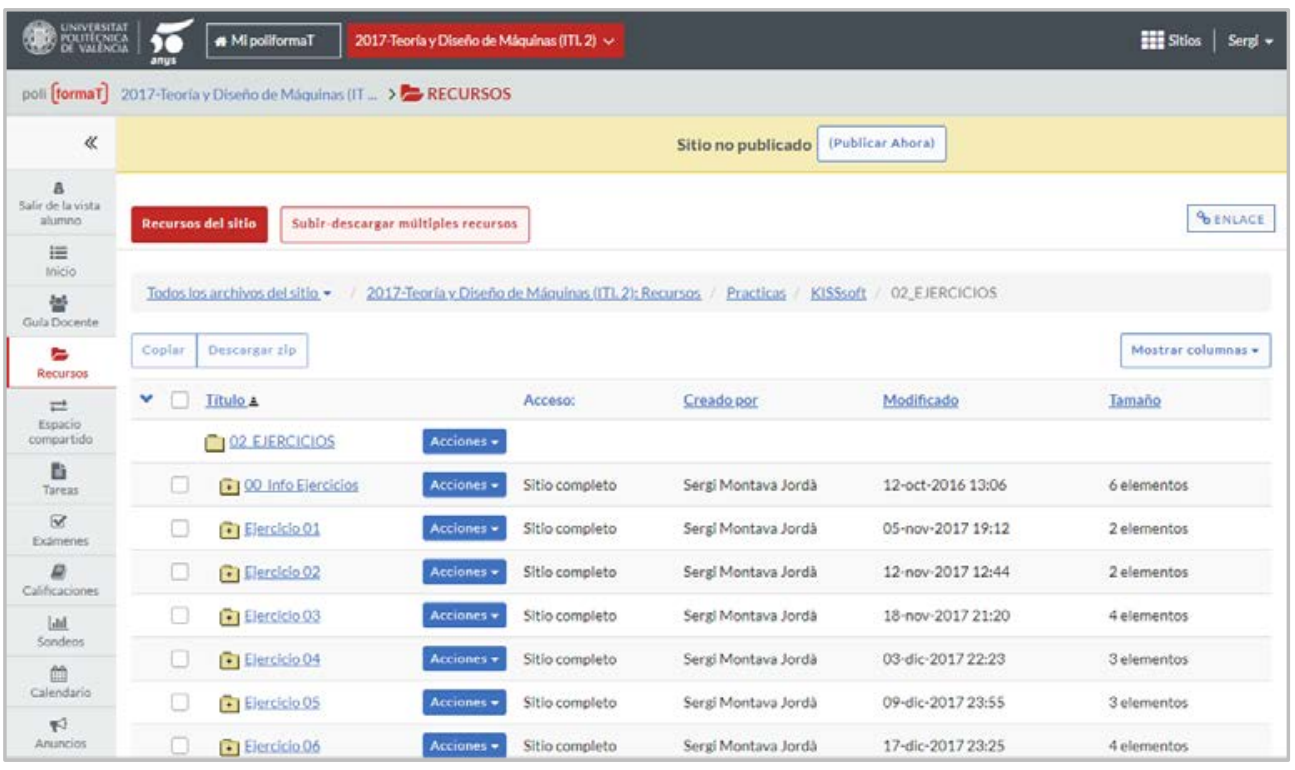

Fig. 2 Apariencia de la carpeta 02_Ejercicios dentro del apartado Recursos del PoliformaT 


\subsection{Pruebas de autoevaluación}

Para poder realizar la autoevaluación del los diferentes ejercicios se han planteado una serie de pruebas auto evaluables a través de la plataforma PoliformaT desde el apartado Exámenes. Estas pruebas están disponibles desde la publicación de los ejercicios el lunes de cada semana que hay una prueba hasta las 20:00 PM del siguiente domingo antes de la siguiente clase. Solamente se permite un envío y el tiempo disponible para su realización es de una hora, tiempo más que suficiente ya que solo se trata de traspasar los resultados a la plataforma.

Las pruebas consisten en un formato de examen de los disponibles en la plataforma PoliformaT en principio con una estructura muy similar todos ellos, en un primer bloque suelen haber un par de preguntas del tipo "Subir ficheros" para que los alumnos puedan subir tanto los archivos del programa especifico como una pequeña práctica en formato pdf y el segundo bloque en el cual hay varias preguntas sobre todo del tipo "Respuesta numérica" para que introduzca un valor que previamente ya tienen realizados.

Desde el apartado exámenes del PoliformaT se puede obtener toda la información al respecto de cada una de las pruebas enviadas por los alumnos aunque en este trabajo nos hemos centrado solamente en las calificaciones, no descartamos para futuros estudios utilizar información como: estadísticas, IP desde la que se realizo la prueba, el tiempo de realización o la fecha de realización desde la publicación del mismo.

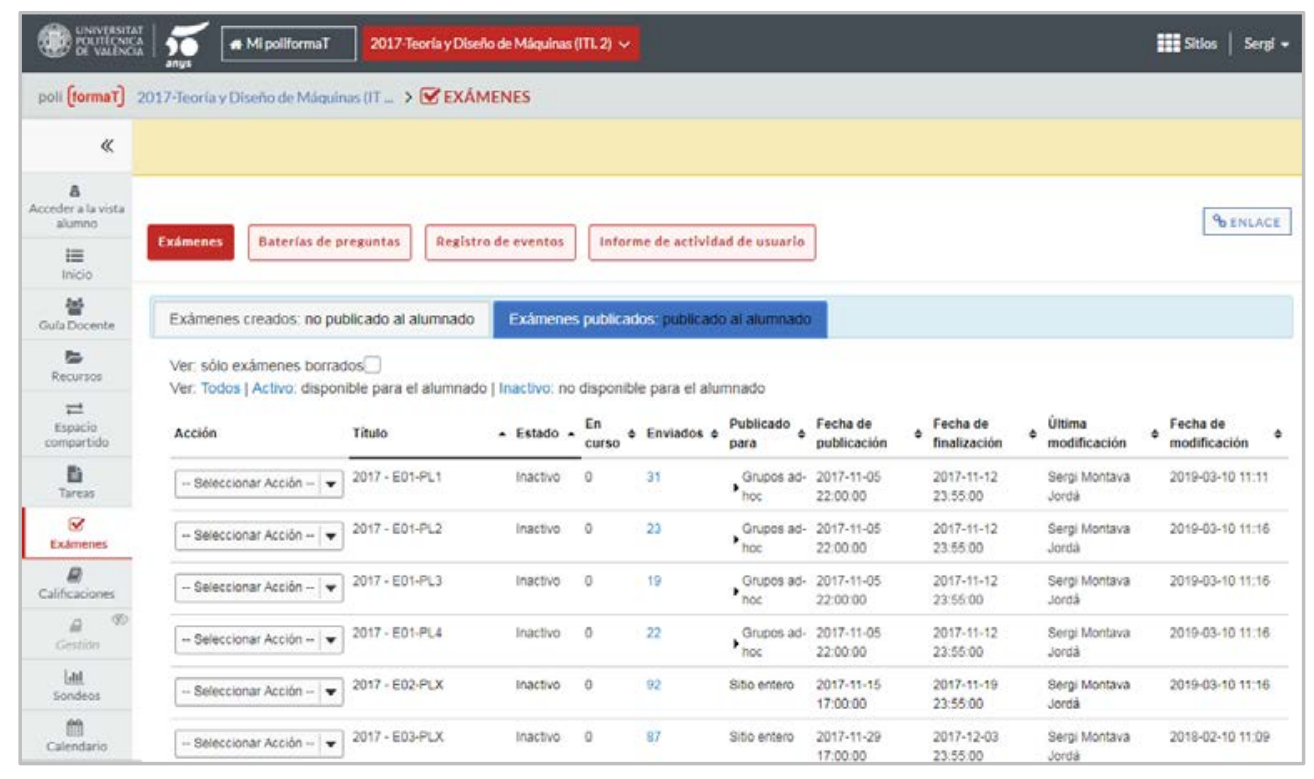

Fig. 3 Apariencia de los Exámenes publicados dentro del apartado Exámenes del PoliformaT 


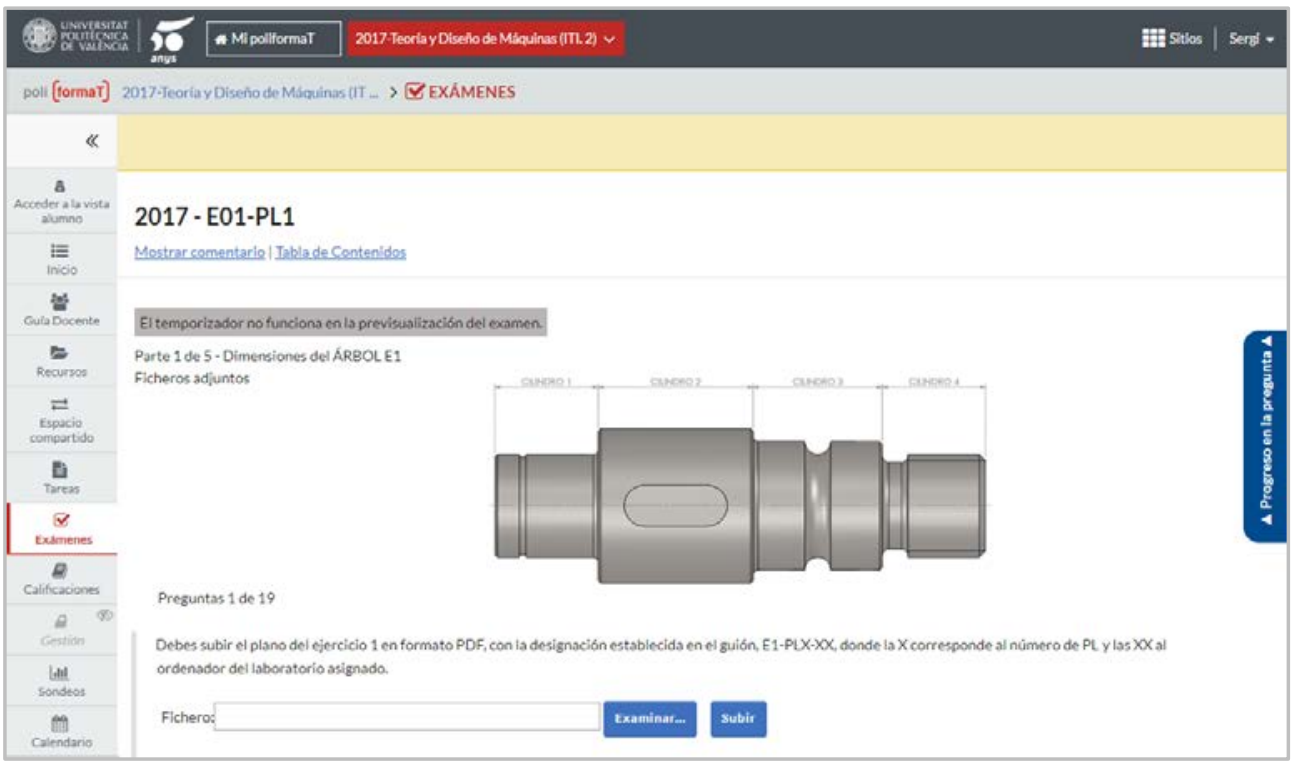

Fig. 4 Vista previa de una prueba auto evaluable dentro del apartado Exámenes del PoliformaT

\section{Resultados}

Al finalizar el curso 2017/2018 se pudieron obtener una serie de datos importantes para poder valorar la iniciativa planteada en este trabajo. En primer lugar, como se puede apreciar en la Figura 5, el nivel de participación no fue el esperado aunque el inicio fue muy bueno, un $75 \%$ de entregados, en los últimos ejercicios el nivel de participación descendió bastante, siendo valor promedio de ejercicios entregados durante todo el curso alrededor del 52\%.

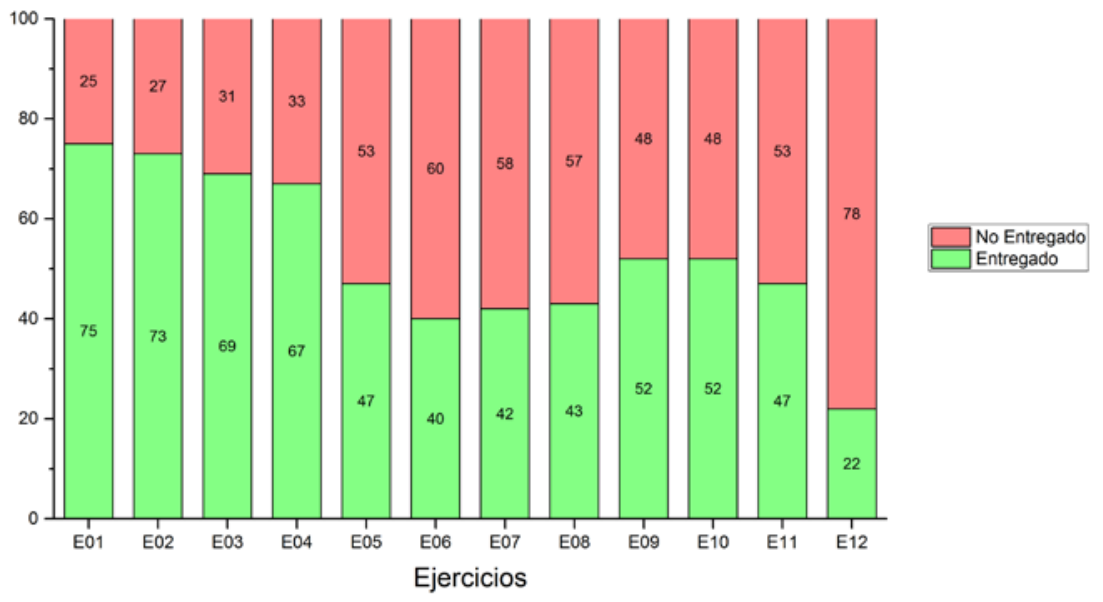

(cc) EY-Nc-ND 2019, Universitat Politècnica de València 
En la Figura 6 se observan cuales han sido la calificaciones del los trabajos de los dos cuatrimestres de los 126 alumnos matriculados durante el curso 2017/2018. El criterio para distribuir el rango de las calificaciones es el siguiente:

- Suspenso, calificaciones inferiores a 5,0 puntos y también los no presentados.

- Aprobado, calificaciones entre 5,0 y 6,9 puntos.

- Notable, calificaciones entre 7,0 y 8,9 puntos.

- Sobresaliente, calificaciones entre 9,0 y 10,0 puntos.

- Matrícula de Honor, es un sobresaliente con mención especial.

En la Figura 7 se representan las calificaciones obtenidas en los dos trabajos de cada curso en porcentaje, ya que la cantidad de alumnos difiere de un curso a otro. Si se incluyen los no presentados como trabajos suspensos, puede apreciarse el porcentaje de suspenso se duplico en el curso 2016/2017 y tras la implantación de la innovación docente se consiguió reducir las calificaciones de Suspenso y Aprobados a costa de un aumento de calificaciones de Notable, Sobresaliente y Matrícula de Honor.

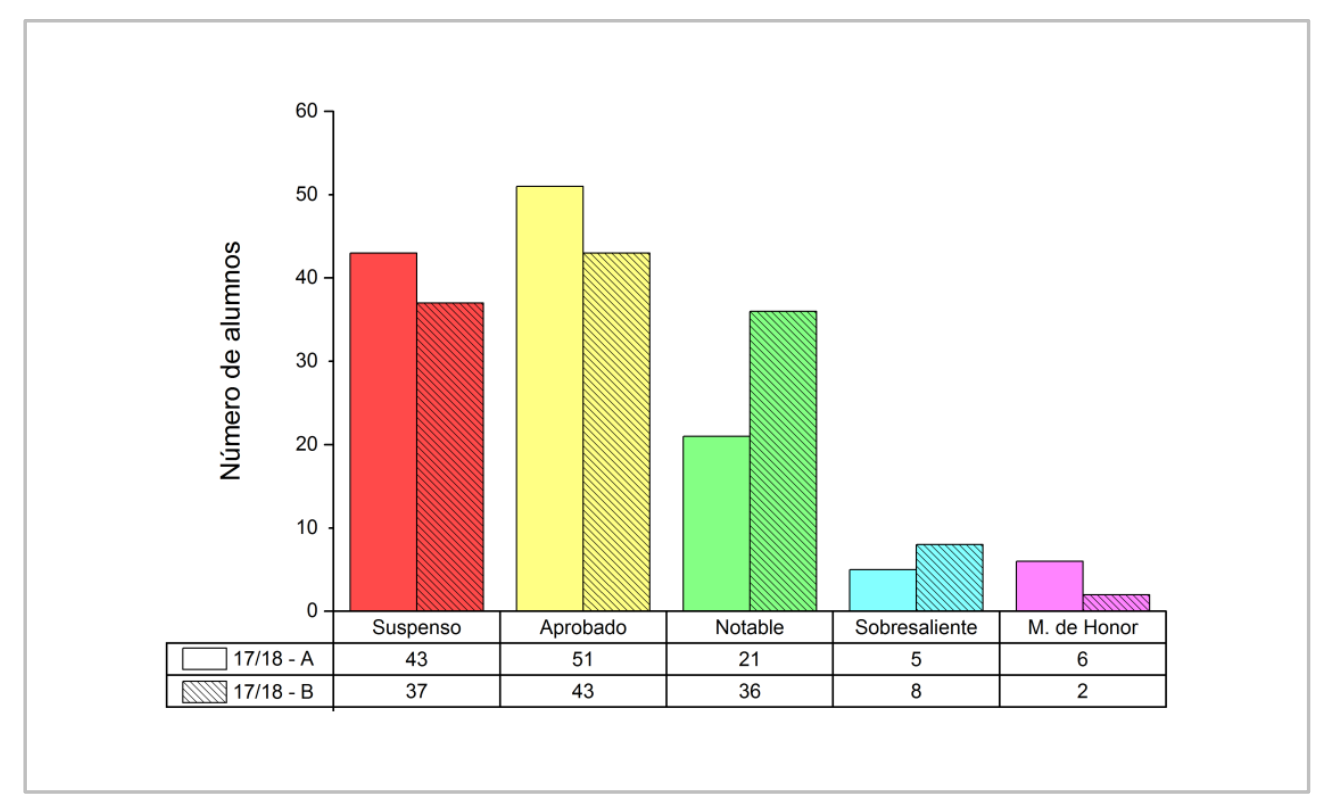

Fig. 6 Calificaciones de los trabajos de prácticas durante el curso 2017/2018 


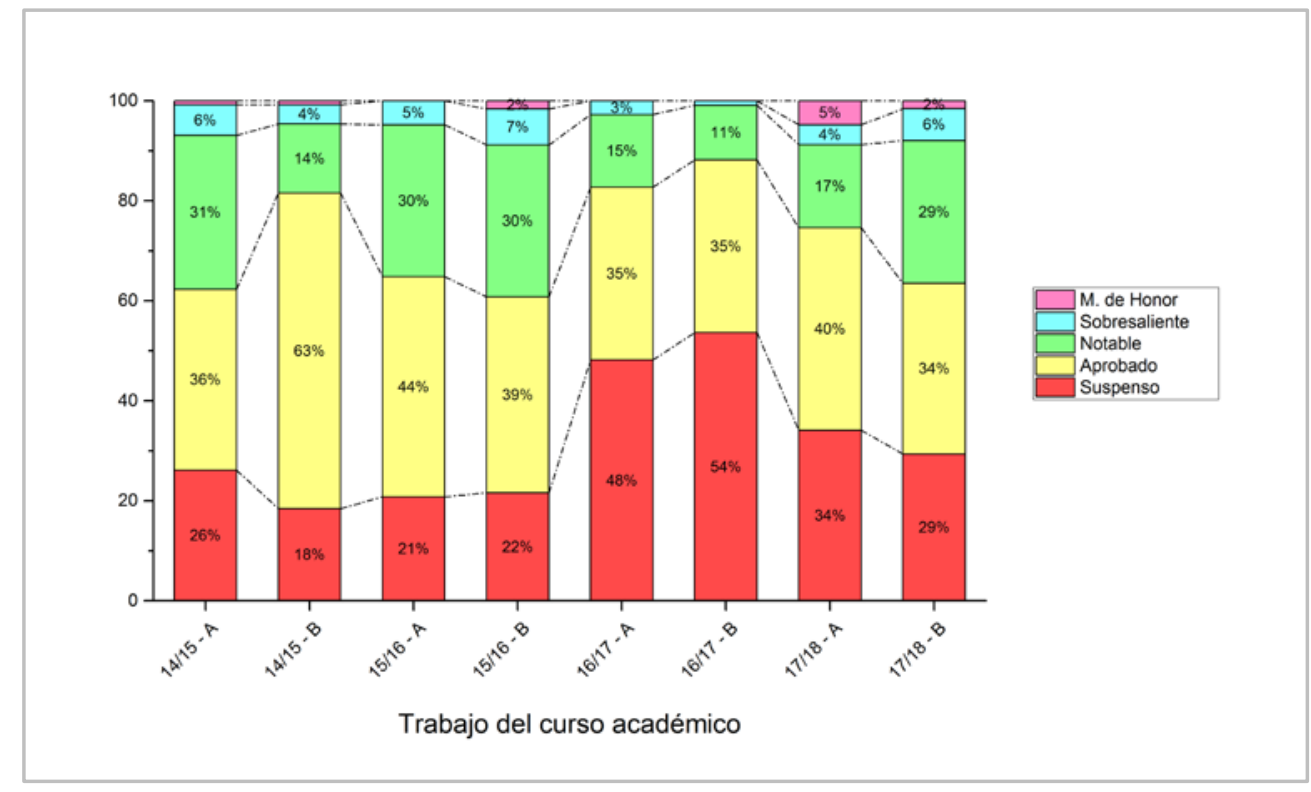

Fig. 7 Evolución de las calificaciones de los trabajos de prácticas en los últimos cursos

Por último cabe destacar que gracias a toda la información que se puede extraer desde la herramienta de Exámenes de PoliformaT se ha podido graficar tanto la nota de los trabajos del cuatrimestre A respecto a la nota media de los ejercicios del E01 al E06, así como la nota de los trabajos del cuatrimestre B respecto a la nota media de los ejercicios del E07 al E12. En la Figura 8 se aprecia una relación tan directa de las notas obtenidas en los ejercicios de autoevaluación respecto a la nota obtenida en los trabajos de prácticas, con lo que queda justificada la satisfacción de los profesores con la iniciativa implantada. 


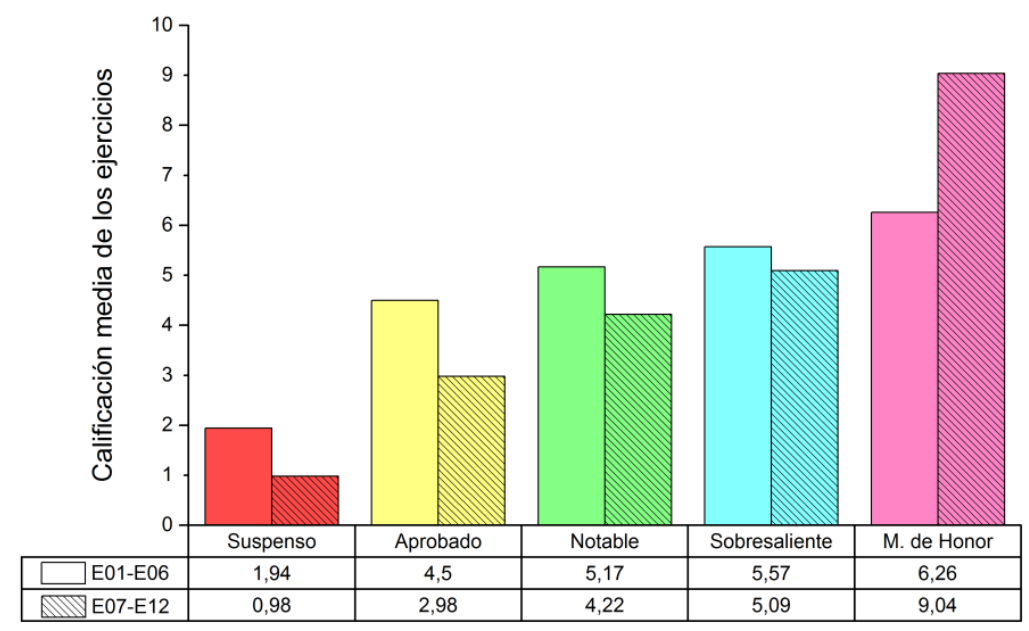

Fig. 8 Relación de la nota media de los ejercicios frente a la de los trabajos durante el curso 2017/2018

\section{Conclusiones}

Los preocupantes niveles en competencias de comprensión, en pensamiento crítico o en la capacidad de resolver problemas con iniciativa que se están detectando en los últimos años, están impulsando al profesorado que utilice nuevas metodologías docentes y en nuestro caso en el ABP reforzado con la metodología activa de autoevaluación. Por otro lado, la implantación del Espacio Europeo de Educación Superior (EEES) en la universidad española implica un cambio en el protagonismo donde se centra en el aprendizaje del alumno.

Aunque ya se ha mencionado con anterioridad cabe destacar que esta metodología ha supuesto una carga de trabajo superior tanto para los alumnos como para los profesores. Pero por otro lado, la realización de estos ejercicios o pruebas semanales auto evaluables aporta al alumno un feedback continuo e inmediato sobre su el trabajo que está realizando, de forma que puede detectar sus equivocaciones o deficiencias en tiempo real sin tener que esperar a la nota del trabajo o examen final pudiendo reaccionar a tiempo para mejorarlas. Además el profesorado puede observar semana a semana cual es la evolución de los alumnos y puede corregir o profundizar más en cuanto aparece alguna dificultad.

La innovación docente de este trabajo, a pesar de ser el primer año y no tener suficiente información para poder contrastarla y sacar conclusiones, pero ha presentado una mejora significante en los resultados de los trabajos de prácticas. El porcentaje de suspensos rondaba el 51\% antes de la implentación y tras esta se ha reducido a un 31,5\%. Aunque la mejora ha podido ser producida por distintas causas como: casualidad, nivel del grupo, trabajos diferentes,... los autores pensamos que la influencia es clara y directa. Por tanto, 
Implementación de las tareas semanales mediante la plataforma Poliformat para la mejora de resultados en el aprendizaje por proyectos.

queda abierta una línea de trabajo futura en la que se realice un seguimiento de los resultados de esta innovación docente.

\section{Referencias}

CODESAL SANABRIA, E. (2008). "Evaluación continua a través del PoliformaT". Paper presented at the VI Jornades d'Investigació en Docencia Universitaria: la construcció col- legiada del model docent universitari del segle XXI.

LEÓN DE MORA, C., MOLINA CANTERO, A. J., MOLINA CANTERO, F. J., \& BISCARRI TRIVIÑO, F. (2009). "Evaluación activa y mejora de la calidad de enseñanza: metodologías e indicadores". Pixel-Bit, 34, 5-12.

LLORENS MOLINA, J. A. (2017). "La evaluación formativa: un análisis desde la perspectiva de los estudiantes". Paper presented at the In-Red 2017. III Congreso Nacional de innovación educativa y de docencia en red.

MORÁN GRACIA, J., \& SEPÚLVEDA PINAR, M. A. (2009). "Una experiencia práctica de evaluación por competencias mediante el uso del portafolio del estudiante y su impacto temporal". Revista de Formación e Innovación Educativa Universitaria, 2(2), 76-86.

RAMÓN FERNÁNDEZ, F. (2011). "La utlización de la Plataforma Poliformat en la enseñanza del Derecho Espacial aplicado a la Ingeniería Aeronáutica". Congreso Internacional de Innovación Docente, Universidad Politécnica de Cartagena, 2011, 23-35.

SÁNCHEZ CABALLERO, S., SELLES CANTÓ, M., PLÁ FERRANDO, R., \& MONTAVA JORDÀ, S. (2018). "Utilización de Wolfram Mathematica la resolución de problemas de Ingeniería". Paper presented at the IN-RED 2018. IV Congreso Nacional de Innovación Educativa y Docencia en Red. 